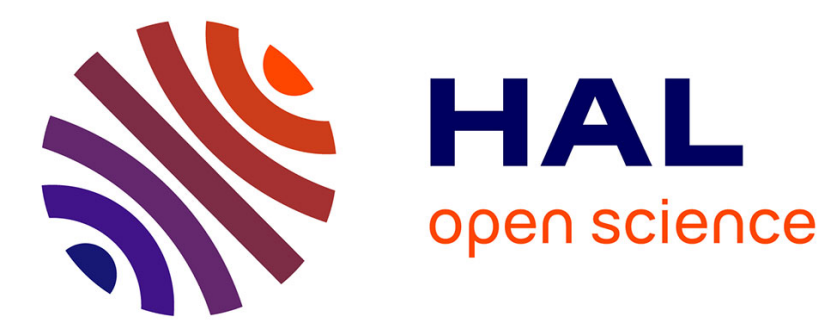

\title{
Répétitions et ritournelles deleuze, les mouvements dansants
}

\author{
Romain Bigé, Paule Gioffredi
}

\section{To cite this version:}

Romain Bigé, Paule Gioffredi. Répétitions et ritournelles deleuze, les mouvements dansants. Repères, cahier de danse, 2015, 35, pp.24-27. 10.3917/reper.035.0024 . hal-03251188

\section{HAL Id: hal-03251188 \\ https://hal.science/hal-03251188}

Submitted on 25 Jun 2021

HAL is a multi-disciplinary open access archive for the deposit and dissemination of scientific research documents, whether they are published or not. The documents may come from teaching and research institutions in France or abroad, or from public or private research centers.
L'archive ouverte pluridisciplinaire HAL, est destinée au dépôt et à la diffusion de documents scientifiques de niveau recherche, publiés ou non, émanant des établissements d'enseignement et de recherche français ou étrangers, des laboratoires publics ou privés. 


\title{
RÉPÉTITIONS ET RITOURNELLES DELEUZE, LES MOUVEMENTS DANSANTS
}

\author{
Romain Bigé, Paule Gioffredi
}

La Briqueterie / CDC du Val-de-Marne | «Repères, cahier de danse »

$2015 / 1 n^{\circ} 35 \mid$ pages 24 à 27

ISSN 2112-5147

Article disponible en ligne à l'adresse :

http://www.cairn.info/revue-reperes-cahier-de-danse-2015-1-page-24.htm

\section{Pour citer cet article :}

Romain Bigé et Paule Gioffredi, « Répétitions et ritournelles deleuze, les mouvements dansants », Repères, cahier de danse 2015/1 (n 35), p. 24-27.

DOI 10.3917/reper.035.0024

Distribution électronique Cairn.info pour La Briqueterie / CDC du Val-de-Marne.

(C) La Briqueterie / CDC du Val-de-Marne. Tous droits réservés pour tous pays.

La reproduction ou représentation de cet article, notamment par photocopie, n'est autorisée que dans les limites des conditions générales d'utilisation du site ou, le cas échéant, des conditions générales de la licence souscrite par votre établissement. Toute autre reproduction ou représentation, en tout ou partie, sous quelque forme et de quelque manière que ce soit, est interdite sauf accord préalable et écrit de l'éditeur, en dehors des cas prévus par la législation en vigueur en France. Il est précisé que son stockage dans une base de données est également interdit. 


\title{
LA RÉPÉTITION ENTRE PHILOSOPHIE ET CHORÉGRAPHIE: LES DANSEURS ET DELEUZE
}

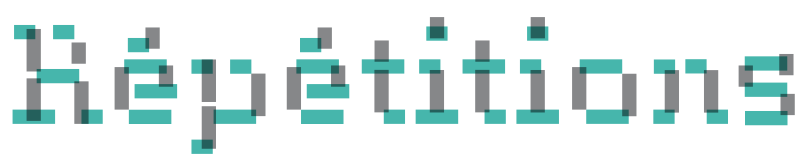 \\ et ritoirjulelles
}

DELEUZE, LES MOUVEMENTS DANSANTS

par Romain Bigé et Paule Gioffredi

ROMAIN BIGÉ, agrégé de philosophie, est actuellement doctorant contractuel à l'École Normale Supérieure. Il y prépare une thèse de philosophie sur le concept de mouvement dans la philosophie du $\mathrm{Xx}^{\mathrm{e}}$ siècle et sur la poétique du Contact Improvisation.

PAULE GIOFFREDI, agrégée de philosophie et auteur d'une thèse d'esthétique intitulée Le porte-̀े-faux: une notion merleau-pontyenne pour penser la danse contemporaine, est maître de conférences au sein du département des arts de la scène, de l'image et de l'écran de Lyon II. Elle est membre du comité de rédaction de la revue Repères, cahier de danse.

LÉGENDE

Répétition de Syndrome amnésique avec fabulations, de Tomeo Vergés, interprété par Julien Lacroix, Sébastien Laurent, Alvaro Morell, Christian Ubl.

Photographie: Patrick Berger.

\section{QUAD: ÉPUISER L'ESPACE}

Deleuze n'est pas seulement sollicité dans les pratiques discursives et artistiques des différents acteurs du milieu de la danse ${ }^{1}$ : il a aussi écrit sur la danse. En 1992, à l'occasion de la publication en français de pièces écrites par Samuel Beckett pour la télévision, il a conçu un texte de quelques pages, au détour d'un commentaire plus large sur le motif de l'épuisement dans le théâtre beckettien, mais bien un texte sur une pièce chorégraphique: Quad ${ }^{2}$, écrite en 1980, réalisée pour la télévision allemande par le dramaturge lui-même en 1981 et publiée l'année suivante en anglais.

Il s'agit d'une pièce sans paroles, une chorégraphie répétitive, minimale, et totale: durant une vingtaine de minutes, quatre personnages parcourent les côtés (longs de six pas) et les diagonales d'un carré, sans jamais aller jusqu'au centre, "supposé zone de danger ${ }^{3}$ ", du quadrilatère, de sorte que «les corps s'évitent respectivement » et «évitent le centre absolument ${ }^{4}$ ». Pour Deleuze, Quad constitue une «ritournelle essentiellement motrice ${ }^{5}$ » et son scénario "est parfaitement clair: il s'agit d'épuiser l'espace $^{6}{ }^{\prime}$. Chaque personnage suit un trajet au cours duquel il sillonne l'ensemble des segments du carré et, par un jeu d'entrées et de sorties, tous les quatre exécutent quatre séries de combinaisons de leurs trajets: "Quatre solos possibles, tous ainsi épuisés. Six duos possibles, tous ainsi épuisés [...]. Quatre trios possibles deux fois, tous ainsi épuisés.» Le projet, dans Quad, serait ainsi d'exténuer la potentialité de l'espace en évitant systématiquement le seul événement possible en lui, à savoir la rencontre, la collision, en son centre, de ceux qui le peuplent. À travers ces quelques minutes de marche ininterrompue, Quad contribue, d'après Deleuze, à «montrer que l'épuisement (exhaustivité) ne va pas 
NOTES

1. Pour une analyse de ces reprises, cf. notam ment R. Huesca, "Ce que fat Deleuz que fait Delouze à danse ", Le Portique n²0, 2007. [http:// leportique.revues. org/1368]

2. S. Beckett, Quad et autres pièces pour la télévision suivi de L’Épuisé par Gilles Deleuze, Minuit, 1992.

3. S. Beckett, Quad, op. cit., p. 14

4. G. Deleuze, L'Épuisé, op. cit., p. 83.

Ibid., p. 81

6. S. Beckett, Quad op. cit., p. 10-11.

7. G. Deleuze, L'Épuisé, op. cit., p. 61.

8. Ibid., p. 83.

9. Ibid, P. 80

o. De même que les références à la danse moderne et populaire dans L'image-temps, se font à partir du cinéma et des problématiques cinématographiques, plutôt que propremen chorégraphiques.

11. D. Lapoujade, Deleuze, les mouvements aberrants, Minuit, 2014.

12. Ibid., p. 9.

13. Ibid., p. 22

14. Ibid p. 18

15. « Pour Deleuze, un mouvement est d'autant plus logique qu'il échappe à toute rationalité. Plus c'est irrationnel, plus c'est abe rant, plus c'est logique rant, plus c'est logique pourtant. »Ibid., p. 13

16. G. Deleuze, Différence et Répétition, PUF, 1996, p. 19.

17. Créé par le Tanztheater Wuppertal le 9 décembre 1978 à l'Opéra de Wuppertal.

18. " "La différence est de rière toute chose, mais derrière la différence, il n'y a rien" [G. Deleuze, Différence et Répétition, op. cit., p. 80].

Il revient à la répétition de briser les cercles par lesquels le fondepar lesquels le fondement se subordonne son action. [.] Elle lui substitue d'] Elle lut cularités, décentrées divergentes, "fondées" sur la différence. C'est ce dont témoigne l'exathèses du temps dans Différence et Répétition qui constituent les trois grands moments de la répétition. Il s'agit en effet, par approfonen effet, par approfondissements successifs, de remonter du prése comme fondation du temps (première synthèse) vers un passé pur comme fondement pur comme fondement
du temps (deuxième synthèse) jusqu'à l'audelà de tout fondement, le pur avenir comme "effondement" (troisième synthèse)." D. Lapoujade, op. cit., p. 49 . sans un certain épuisement physiologique », "grand apport de Beckett à la logique ${ }^{7}$ ».

En cela, Deleuze considère que «Quad est proche d'un ballet » et que "les concordances générales de l'œuvre de Beckett avec le ballet moderne sont nombreuses ». En effet, précise-t-il, les corps des interprètes de Quad, totalement recouverts par de larges toges, ne s'exposent pas érigés, virtuoses, en gloire, mais font voir et entendre leurs déplacements, et ces allées et venues sur un pla teau vide et obscur ne tissent aucun récit, n'évoquent aucun contexte qualifié, mais déploient une pure «logique de postures et de positions ${ }^{8}$ » dans un «espace quelconque mais défini » (un carré, quelconque; mais un carré $^{9}$, défini).

Cet intérêt de Deleuze pour une danse, certes circons tancié à l'œuvre d'un homme de théâtre ${ }^{10}$, n'est toutefois pas fortuit. L'œuvre toute entière du philosophe tend à s'emparer de la question du mouvement, comme le montre avec justesse David Lapoujade dans son récent ouvrage: Deleuze, les mouvements aberrants ${ }^{11}$. Pour ce dernier, "la philosophie de Deleuze se présente comme une philosophie des mouvements aberrants ou des mou vements “forcés" 12 ", c'est-à-dire qui nous arrachent à nous-mêmes, "que nous ne pouvons vivre qu’à la limite de nous-mêmes", qui font "qu'on ne tient plus à sa vie dans ce qu'elle a de personnel, mais à l'impersonne qu'elle permet d'atteindre, de voir, de créer, de sentir à travers elle ${ }^{13}$ ». Dès lors, ces mouvements "débordent tout vécu » et traversent pourtant l'existence pour l'ouvrir et la porter à avancer, ils forcent chaque faculté «à se dépasser vers un objet qui la concerne exclusivement, mais qu'elle n'atteint qu'à la limite d'elle-même ${ }^{14}$ ». Deleuze aurait été hanté par le problème d'établir leur existence, de les répertorier et de définir la logique (non rationnelle ${ }^{15}$ ) à laquelle ils obéissent pour cerner leurs raisons d'être.

\section{LE THÉÂTRE DE LA RÉPÉTITION}

Tel est, nous semble-t-il, un des points de départ du premier grand livre de Deleuze (sa thèse, publiée en 1968), où le philosophe se propose d'enquêter sur ces mouvements que sont Différence et Répétition. Deleuze pose d'abord la question de savoir ce qu'il y a d'aberrant dans la répétition: quelle sorte de plaisir, d'intérêt, de force peut-on bien trouver dans le ressassement d'une même idée ou d'un même schème moteur? Qu'est-ce qui s'y éprouve (sensation et épreuve)?

Le premier geste de Deleuze pour répondre à cette question est de réfléchir au «théâtre» sur lequel cette opération se produit, théâtre qui n'est sans doute autre que cet «espace épuisé » de Quad:

«Dans le théâtre de la répétition, on éprouve des forces pures, des tracés dynamiques dans l'espace qui agissent sur l'esprit sans intermédiaire, et qui l'unissent directement à la nature et à l'histoire, un langage qui parle avant les mots, des gestes qui s'élaborent avant les corps organisés ${ }^{16}$.»

Ce théâtre de la répétition, Deleuze l'oppose au théâtre de la «représentation". Ou plus exactement: la répéti tion, c'est le mouvement aberrant de la représentation. Qu'est-ce à dire? La logique de la représentation, c'est la logique qui veut que l'on passe d'un modèle (texte, chorégraphie, idée) à son imitation ou au mieux à son inter prétation sur scène. Au contraire, la logique de la répétition veut que paradoxalement rien n'y soit repris. C'est là une des grandes idées défendues par Deleuze dans
Différence et Répétition: on ne répète rien. Non pas seulement parce qu'"on ne se baigne jamais deux fois dans le même fleuve", mais surtout parce qu'en toute rigueur, on ne sait jamais vraiment quand a commencé la série des répétitions. Au cours de la création d'un spectacle, à quel moment passe-t-on de l'invention à la reprise? En anglais, la répétition de théâtre se dit rehearsal: étymologiquement, cela signifie repasser la herse, creuser des sillons. Mais quelle était la première ligne qu'on a suivie pour les creuser? Comme le note Brigitte Asselineau (voir p. 3): «Il n'y a pas vraiment de mots pour l'avant de la répétition. On pourrait dire "j'entre en création" mais je l'entends rarement; il est assez étonnant que l'on parle de ce temps, déjà, comme d'une répétition...»

Pour laisser cette question en suspens, Deleuze reprend à la psychanalyse l'idée d'une série qui a "toujours déjà » commencé; elle n'a pas de terme ultime, elle n'a pas de point de départ. Toutes les compulsions (vérifier les portes bien fermées), tous les amours qui reprennent le premier (aimer, comme Descartes, les femmes qui louchent) relèvent de ce bizarre paradoxe: je ne répète pas parce que j'ai oublié que je l'avais déjà fait; j'oublie que je l'avais déjà fait parce que je répète (je recouvre la première fois du voile de sa copie). Il n'y a rien qui soit répété (il n'y a pas d'originaire effectif) parce que la répétition est justement la suppression (l'oubli) de ce premier terme. C'est pourquoi la répétition n'est jamais une re-présentation, un retour, mais le contraire d'un retour puisqu'elle supprime ce qui serait susceptible de revenir. Deleuze nomme cela, dans la danse de Quad, «épuiser l'espace », c'est-à-dire manifester l'impossibilité du retour sur les mêmes trajets.

Deleuze soulève ici un des paradoxes majeurs de la répétition: en même temps qu'elle permet de s'approprier un schème moteur (par fixation de l'habitude), elle le fait toujours au risque d'en annuler le contenu pour n'en conserver que la forme extérieure. C'est ce matériau que travaille Pina Bausch dans la scène de Kontakthof ${ }^{17}$ où Nazareth Panadero se fait manipuler par un groupe d'hommes qui la caressent légèrement, par de petits attouchements sur chacune des parties de son corps (cheveux, front, genoux, joues, oreilles, cou, jambes, pieds, nez, hanches, etc.). À force de répétition, ces gestes d'attention se transforment en gestes de possession, Nazareth Panadero devient l'objet d'une lutte entre les hommes: le retour incessant des caresses les vide de leur contenu intentionnel; on ne voit plus que la prolifération d'un mouvement qui envahit le corps de l'autre.

\section{LES TROIS SYNTHÈSES DU TEMPS POUR PENSER LE DANSER}

Le cœur de Différence et Répétition est consacré à une longue démonstration sur la genèse de la temporalité. Le philosophe explique que, loin d'avoir lieu dans un temps qui lui préexisterait, la répétition engendre le temps. Il repère trois modalités de cette temporalisation: la répétition de l'habitude qui constitue le présent, la répétition de la mémoire qui constitue le passé, et enfin l'événement qui ouvre sur l'avenir de ses répercussions. David Lapoujade précise que ces synthèses ou modes de temporalisation se dépassent réciproquement dans une marche vers le "sans-fond ${ }^{18}$ », c'est-à-dire vers cela même que les mouvements aberrants expriment et manifestent. En nous appuyant sur les témoignages d'artistes de danse, tout particulièrement ceux qui ont participé à ce numéro de la revue Repères, cahier de danse, et sur la > 
NOTES

19. W. Piollet, Aventure des barres flexibles, L'une \& l'autre, 2014, p. 19

2o.D. Lapoujade, op. cit., p. 65 .

21. P. Bausch citée par D. Frétard, «Pina

Bausch, l'exorciste »

Le Monde, 10 fév. 1995

22. D. Lapoujade,

op. cit., p. 70.

23.Ibid., p. 71.

24. "Au-delà de la répétition nue et de la répétition vêtue, au-delà d celle à laquelle on soutire la différence et de celle qui la comprend, une répétition qui "fait" la différence. Au-delà de la répétition fondé et de la fondatrice, une répétition d'effondement dont dépendent à la fois ce qui enchaîne et ce qui libère, ce qui meurt et ce qui vit dans la répétition.» G. Deleuze, Différence et Répétition, op. cit., p. 374. 25. D. Lapoujade, op. cit., p. 85

LÉGENDE

Anne-Lise Le Gac et

Élie Ortis en répétition

dans le studio de

la Compagnie

Ultima Vez /

Wim Vandekeybus

(Bruxelles).

๑ GRAND MAL
> relecture à laquelle David Lapoujade invite à la lumière de la notion de mouvements aberrants, nous aimerions indiquer comment la théorie deleuzienne des syn thèses du temps peut aider à penser certains des enjeux propres à la vie artistique des danseurs. Nous nous arrêterons sur trois modalités de la répétition du danser: le travail de l'habitude dans la formation et l'entraînemen quotidien, l'intervention d'une forme de mémoire dans le processus de création, et le retour de l'événement lors des représentations d'une œuvre.

Wilfride Piollet explique dans son Aventure des barres flexibles que l'échauffement, l'entraînement quotidien est une partie capitale de la vie du danseur car «c'est à cette occasion qu'il se construit, qu'il se découvre, qu'il forge les outils qui lui permettront de s'incarner dans ses mouvements ${ }^{19} »$. Pour reprendre le vocabulaire deleuzien, la répétition de mouvements habituels construit progressivement un territoire, mais ce dernier se donne comme un domaine à explorer et une base, une forme de foyer d'où partir à l'aventure. En cela, les répétitions de l'habitude sont bien "fondation du temps ${ }^{20}$ »: elles sont, comme souligne Brigitte Asselineau, un travail de "recherche » et d'«humilité » au cours duquel il s'agirait de "ne pas se contenter de ce que l'on sait faire», d'«accepter de répéter pour aller plus loin». Cette première synthèse n'est donc pas seulement génératrice d'un territoire, mais se double de l'émergence d'une virtualité et du désir d'en expérimenter la richesse. Ce dont témoigne Anne-Lise Le Gac, qui va jusqu'à concevoir l'entraînement à la fois comme pratique et comme matière pour composer une performance.

Dans cette perspective, l'originalité et la force d'une œuvre chorégraphique tiendrait à la capacité des artistes qui s'y engagent à découvrir ce que leur rencontre dans leur situation permet de (re)trouver qui à la fois soit propre à ces circonstances particulières et en même temps commun à tous ceux et tout ce qu'elle implique et concerne, public compris. Cécile Léonardi nous a rappelé par exemple que Pina Bausch fit l'expérience, au cœur du singulier contexte de la création de Viktor et de la construction d'une figure expressive du deuil, de ce surgissement d'un geste archétypal: «Dans Viktor, en signe de deuil, nous avions trouvé le geste qui consistait à se frapper la tête avec ses poings. Ce geste existe en fait dans toutes les civilisations. On invente moins qu'on ne le croit. On retrouve ${ }^{21}$.» Ainsi, au cours d'un processus de création, la justesse d'une proposition serait évaluée à l'aune de critères propres au projet en cours, qui ne résultent ni de la simple reprise du connu et reconnu, ni d'une invention pure, mais précisément de l'émergence d'un possible déjà là mais que tout concourt, ici et maintenant, à actualiser et expérimenter: «chaque présent est une répétition de notre passé tout entier, contracté sous tel ou tel aspect ${ }^{22}$ ", écrit David Lapoujade, de sorte qu' "il y a comme une échelle élective en fonction de laquelle sont placés au sommet les présents les plus contractés, ceux qui contractent la plus grande part de notre passé et, au bas de l'échelle au contraire, les présents les plus relâchés ${ }^{23}$ ». Paradoxe que Laurent Pichaud pousse à son comble quand il intervient dans l'espace public et, de ce fait, renonce à la distinction de la répétition, du lieu et du moment de préparation, et de la représentation, de ses contours et de ses occurrences: il porte à son point culminant la synthèse du passé dans le singulier, à la fois déterminé et ouvert, lisse et strié, du contexte.

Enfin, les analyses concernant la synthèse de l'avenir, dimension du temps que Deleuze nomme aussi "effondement $^{24}$ ", permettraient de penser l'itération de l'interprétation d'une pièce: au fil des jours et au gré des lieux, plutôt que d'exécuter leurs mouvements, les danseurs tenteraient, à chaque fois, de retrouver le vivant, l'ouvert, de l'aberrant plutôt que de l'automatique et ainsi d'œuvrer à la «redistribution permanente de puissances du sans-fond ${ }^{25}$ ». Ainsi, par exemple, Tomeo Vergés insiste sur l'écueil de la robotisation du mouvement et de la sclérose de la danse dans la raideur d'une interprétation qui ne serait plus animée. Pour lui, chaque reprise et représentation exige de "re-demander», pour "être actif de nouveau» et demeurer suffisamment vigilant pour «laisser traverser le vivant». De cette manière, chaque occurrence d'une pièce pourrait être conçue, 
NOTES

26.Ibid., p. 79 .

27. G. Deleuze et

F. Guattari, Mille

Plateaux, Minuit, p. 38

28. Créé par les Ballets

C. de la B., en janvier

2004, au Münchner

Kammerspiele.

29. M. Proust, À la

recherche du temps

perdu, Gallimard,

1988 , t. II, p. 387.

30.G. Deleuze, Différence

et Répétition,

op. cit., p. 13.

31. G. Deleuze et

F. Guattari, op.

cit., p. 382-383.

32. Créé avec le San

Francisco Dancers

Workshop à Stockholm

le 5 septembre 1965 .

33. A. Halprin, Collected

Writings, San Francisco

(CA), San Francisco

Dancers Workshop,

1974, p. 1-2.

34. A. Damasio, La Horde

du Contrevent,

Folio, 2014, p. 543 selon les termes de David Lapoujade, "comme une diagonale qui coupe le temps chronologique [...], introdui sant en lui l'avant et l'après ${ }^{26}$ ", ce que Deleuze nomme un événement.

À nos yeux donc, le commentaire des synthèses temporelles par David Lapoujade contribue à articuler la philosophie deleuzienne à la danse via la répétition en montrant que cette dernière, en tant qu'épreuve de la différence, permet d'accéder aux mouvements aberrants. L'art chorégraphique pourrait être conçu comme une surface adéquate au travail, caractéristique des mouvements aberrants, de perforation ou d'épuisement de la représentation. La répétition s'y avérerait différenciation et l'itération événement susceptible de redistribuer les puissances de la vie.

\section{LA RITOURNELLE: CHEZ-SOI ET ÉCHAPPÉE}

Dans le onzième des Mille Plateaux, consacré à «la ritournelle », Deleuze et Guattari proposent une porte de sortie à la sclérose de la répétition : un mouvement qui se laisserait contaminer par ses propres forces centrifuges (comme une ronde qui, à force de tourner, éjecterait ses danseurs hors du cercle). L'idée de Deleuze et Guattari est que ce n'est pas en ajoutant un nouvel élément à ce que nous répétons que nous parvenons à sortir du cercle: c'est à même la reprise incessante du même geste qu'on finit par s'échapper. Bref, pour sortir de ses habitudes, il faut se les approprier, ou, plus profondément, pour pouvoir sortir de chez soi, il faut le constituer, et telle est la fonction des deux premiers aspects de ce que Deleuze et Guattari appellent les «ritournelles territoriales».

Premier aspect: face à l'incertitude, se rassurer en esquissant "un centre stable et calme, stabilisant et calmant, au sein du chaos ${ }^{27}$ ». Deleuze et Guattari prennent l'exemple de l'enfant seul dans le noir entamant une chansonnette pour se tenir compagnie avec du déjà connu. Qu'on pense à la clameur des gestes et des cris de «Papillon! » lancés par Romeu Roma dans Tauberbach ${ }^{28}$ d'Alain Platel: face au chaos des vêtements, ces peaux d'homme, amoncelées sur le plateau, Romeu Roma rejoue, sans cesse, son entrée en Papillon; il semble dire "au moins, ça, le chaos ne me le volera pas». Quand j'appréhende un nouveau milieu, mais aussi bien quand j'apprends du nouveau (un nouveau style, un nouveau chorégraphe, une nouvelle partition), je m'efforce d'y insérer mes petites ritournelles, mes petites habitudes, de colorer ce milieu d'un peu de connu, pour ne pas y être complètement perdu.

Deuxième aspect: une fois chez soi, constamment confir mer ce qui m'est propre, affirmer et ré-affirmer que cela, du moins, je le connais ou sais le faire. Ici, la répétition permet de faire le tri. Cette ritournelle d'appropriation du chez-soi concerne par exemple nos petites habitudes: petits exercices matinaux, rangements ou réarrangements, tics de langage. Ainsi confirme-t-on ce fait miraculeux, nommé par Proust, de se réveiller, tous les jours, le même que la veille:

"On ne voit pas ce qui dicte le choix et pourquoi, entre les millions d'êtres humains qu'on pourrait être, c'est sur celui qu'on était la veille qu'on met juste la main. Qu'est-ce qui nous guide, quand il y a eu vraiment interruption (soit que le sommeil ait été complet, ou les rêves entièrement différents de nous ${ }^{29}$ )? 》 La deuxième ritournelle est cette opération par laquelle je vérifie que c'est bien moi qui suis là dans ce corps et dans cet environnement. Mais le risque, dans cette ritournelle d'auto-confirmation, c'est qu'elle s'effectue toujours à la limite d'un enfermement: au lieu de répéter, c'est-à-dire de se re-mettre en jeu sans cesse, on ressasse (symptôme ultime de la dépression: quand la répétition devient ressassement). Telle est la question fondamentale qu'adresse Deleuze à la répétition:

«Faire de la répétition même quelque chose de nouveau [...], agir, faire de la répétition comme telle une nouveauté, c'est-à-dire une liberté et une tâche de la liberté $^{30}$.»

Une partie de la réponse à cette question est fournie par le troisième aspect de la ritournelle, qu'on pourrait appeler ritournelle de déterritorialisation. C'est la ritournelle qui, par les mêmes opérations qui constituent le chez-soi, nous fait sortir de son plan:

«Maintenant, enfin, on entrouvre le cercle, on l'ouvre, on laisse entrer quelqu'un, on appelle quelqu'un, ou bien l'on va soi-même au dehors, on s'élance. Et cette fois, c'est pour rejoindre des forces de l'avenir, des forces cosmiques. On s'élance, on risque une improvisation ${ }^{31}$.»

À force de tourner en rond, donc, on s'expulse au dehors ou on invite le dehors à soi: on se risque au regard du spectateur, qu'on ignorait jusque-là; ou bien, on trouve de nouveaux «trucs» pour gagner un centième de seconde ici, une amplitude de mouvement là. Et il est essentiel ici de dire qu'on n'aurait pas trouvé le dehors (en tout cas pas celui-ci) sans utiliser les forces qui nous ont permis de construire le "dedans». Rien de pire, de plus prévisible ou de plus répétitif au sens faible, que la «pure» improvisation («allez-y: dansez!») qui ne s'assoit pas sur un certain chez-soi qu'on se laisse le temps de s'approprier.

C'était déjà la fonction des tâches dans les chorégraphies qu'Anna Halprin concevait dans les années 1960: il s'agissait de fournir un cadre dans lequel la répétition de la même activité (s'habiller / se déshabiller, par exemple, dans Parades and Changes ${ }^{32}$ ) à la fois "vide le mouvement de certains types d'introspection", et ouvre sur «un théâtre où tout est vécu comme si c'était la première fois, un théâtre de risque, de spontanéité, de mise en danger de soi et d'intensité ${ }^{33}$ ".

La troisième ritournelle n'a ainsi pas pour fonction de briser le chez-soi, mais bien d'y faire surgir une forme de vulnérabilité aux forces qui le traversent. Toute la finesse de la proposition de Deleuze consiste donc à dire qu'il ne s'agit pas tant d'éviter la répétition à tout prix, que de trouver, au sein de cet étrange éternel retour du même, ce qui crée et ouvre des différences.

Dans son ouvrage de science-fiction La Horde du Contrevent, Alain Damasio propose une compréhension profonde de cette troisième ritournelle. Il la décrit comme une dimension extra-spatiale et extra-temporelle de la vitesse:

«Le vif n'est pas relatif à un espace ou à une durée. Il n'opère pas un pli ou une déchirure dans un tissu préexistant, comme l'opère le mouvement. Il est le surgissement absolu. Il amène, dans un vent, dans une vie, dans une pensée, le plus petit écart. Un minuscule apport, à peine un grain, et tout explose ${ }^{34}$.» L'intempestif du vif ne signale donc pas une différence inassignable mais une manière de différer au sein même de l'identique. C'est cette foudre de l'instant que, paradoxalement, la troisième ritournelle, par la répétition, cherche à convoquer.

- 